\title{
Bronchiolitis Obliterans Organising Pneumonia (BOOP) in a lung cancer patient after lobectomy
}

\author{
S.W. Yan1, C.F. Wong1, P.C. Wong1 ${ }^{1}$, C.F. Cheung ${ }^{2}$
}

ABSTRACT: Bronchiolitis Obliterans Organising Pneumonia (BOOP) in a lung cancer patient after lobectomy. S.W. Yan, C.F. Wong, P.C. Wong, C.F. Cheung.

A 79 year-old patient with lung cancer underwent a standard thoracotomy and lobectomy. Postoperatively, he developed low-grade fever and dyspnoea. Chest $\mathrm{X}$-rays showed pro- gressive lung infiltrates, which was subsequently diagnosed to be Bronchiolitis Obliterans Organizing Pneumonia (BOOP) by transbronchial lung biopsy. He responded well to corticosteroid therapy. The case report is followed by a brief discussion on BOOP in association with lung cancer and thoracotomy. Monaldi Arch Chest Dis 2005; 63: 1, 55-58.

Keywords: Bronchiolitis Obliterans Organizing pneumonia (BOOP), Lung cancer, Thoracotomy.

1 Tuberculosis and Chest Unit, Grantham Hospital, 125 Wong Chuk Hang Road, Aberdeen, Hong Kong

2 Department of Medicine and Geriatric, Tuen Mun Hospital, Tsing Chung Koon Road, Tuen Mun, Hong Kong, China.

Correspondence: Dr. C.F. Wong, Tuberculosis and Chest Unit, Grantham Hospital, 125 Wong Chuk Hang Road, Aberdeen, Hong Kong, China; e-mail:wongcf2001@yahoo.com.hk

\section{Introduction}

Bronchiolitis Obliterans Organising Pneumonia (BOOP) was first reported by Epler in the 1980s as a clinico-pathological entity [1]. It is characterised by a variable clinical and radiographic picture but a distinct pathological appearance together with a dramatic response to corticosteroid therapy. BOOP has been reported to be associated with malignancy and its treatment [2]. However, in the majority of cases it seemed to be either a silent pathological process in the vicinity of a tumour or an active symptomatic disease secondary to anti-cancer therapy like radiotherapy and /or chemotherapy. Here we report a case of BOOP occurring during the immediate post-operative period in a patient with lung cancer without prior chemotherapy or radiotherapy.

\section{Case Report}

A 79 year-old male patient, with a history of hypertension and diabetes mellitus presented with incidental chest X-ray finding of a right lower lobe mass (figure 1). Computed tomography (CT) of thorax revealed a contrast enhanced spiculated mass measuring $3.5 \mathrm{~cm} \times 6.1 \mathrm{~cm}$ in the right lower lobe. No mediastinal lymphadenopathy or other lung parenchymal abnormalities was noted. Cytological examination of fine needle lung aspirate under CT guidance showed cells suspicious of malignancy. A thoracotomy and right lower lobectomy was performed. A histological examination of the resected right lower lobe showed adenosquamous carcinoma with clear resection margin and the lymph nodes in the resected lung block were also free of malignant involvement.

The patient had been well before the operation with no respiratory symptoms or flu-like illness. Post-operatively, starting from day 5, there was an intermittent low-grade fever of $37^{\circ} \mathrm{C}$ to $38^{\circ} \mathrm{C}$. The

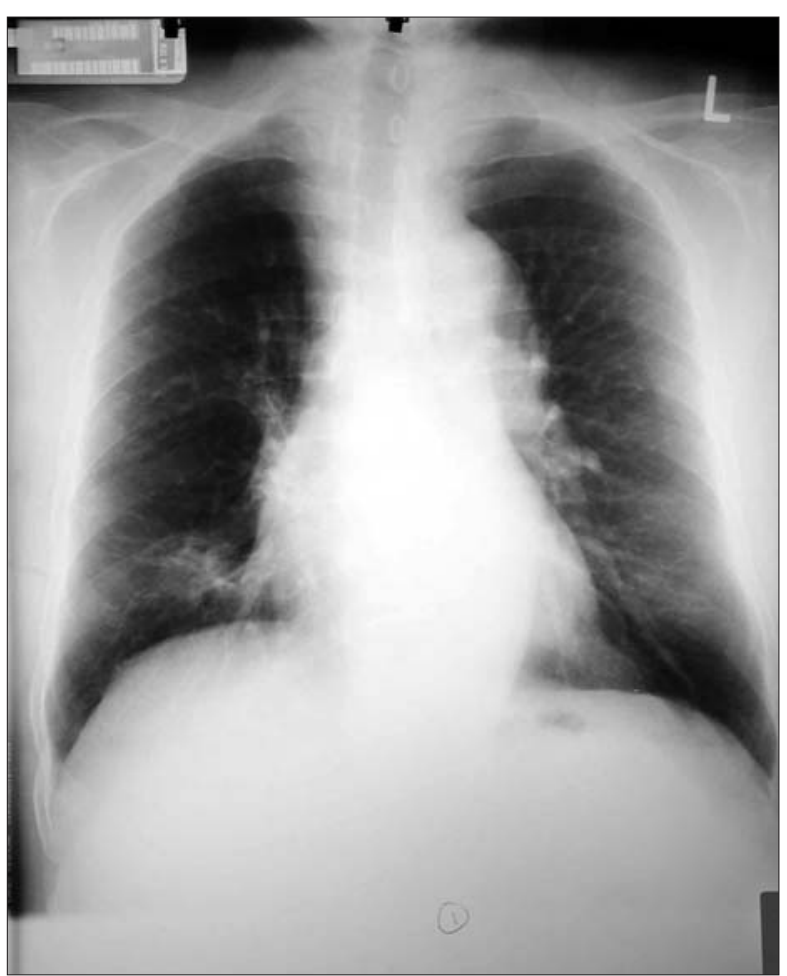

Fig. 1. - Chest radiograph at presentation showing the right lower lobe tumour. 
patient also noticed dyspnoea on exertion but there was only mild and unproductive cough. He was otherwise well and ambulatory. His oxygen saturation was maintained at around $96 \%$ to $99 \%$ on room air at rest. Chest auscultation revealed decreased breath sound and fine inspiratory crepitations at the right lung base. There were moderate systemic leucocytosis (WBC 14.3 x 109/L with neutrophil predominance, $\left.10.7 \times 10^{9} / \mathrm{L}\right)$ and high ESR ( $92 \mathrm{~mm} / \mathrm{hr})$. Chest x-ray showed new alveolar shadows at the right lower zone (figure 2). Sputum examination was negative for bacterial culture and acid-fast bacilli on microscopy. He was treated as a post-operative pneumonia with one week of Cefuroxime followed by one week of Cefoperazone-Sulbactam. However, there was no clinical or radiological response to antibiotic therapy. Apart from the persistence of low-grade fever and dyspnoea, the patient was otherwise well and nontoxic. Blood picture showed persistent high white cell count and ESR of $11.6 \times 109 / \mathrm{L}$ and $100 \mathrm{~mm} / \mathrm{hr}$ respectively. Subsequent chest X-rays showed progression of infiltrates to right middle zone and left lower zone as well (figure 3). CT thorax was done and it showed coarse reticular infiltrates at right lung as well as left lung base with small amount of right pleural effusion. No mediastinal or hilar lymphadenopathy was evident. Lung function tests showed a restrictive defect with $\mathrm{FEV}_{1}$ of $1.15 \mathrm{~L}$ (54\% predicted) and FVC of $1.40 \mathrm{~L}$ (50\% predicted). Blood for immune markers were negative. Fibre-optic bronchoscopy was carried out on postoperative day 35 and it showed right lower lobe post-lobectomy changes with normal looking stump. Bronchial aspirate was negative for malig-

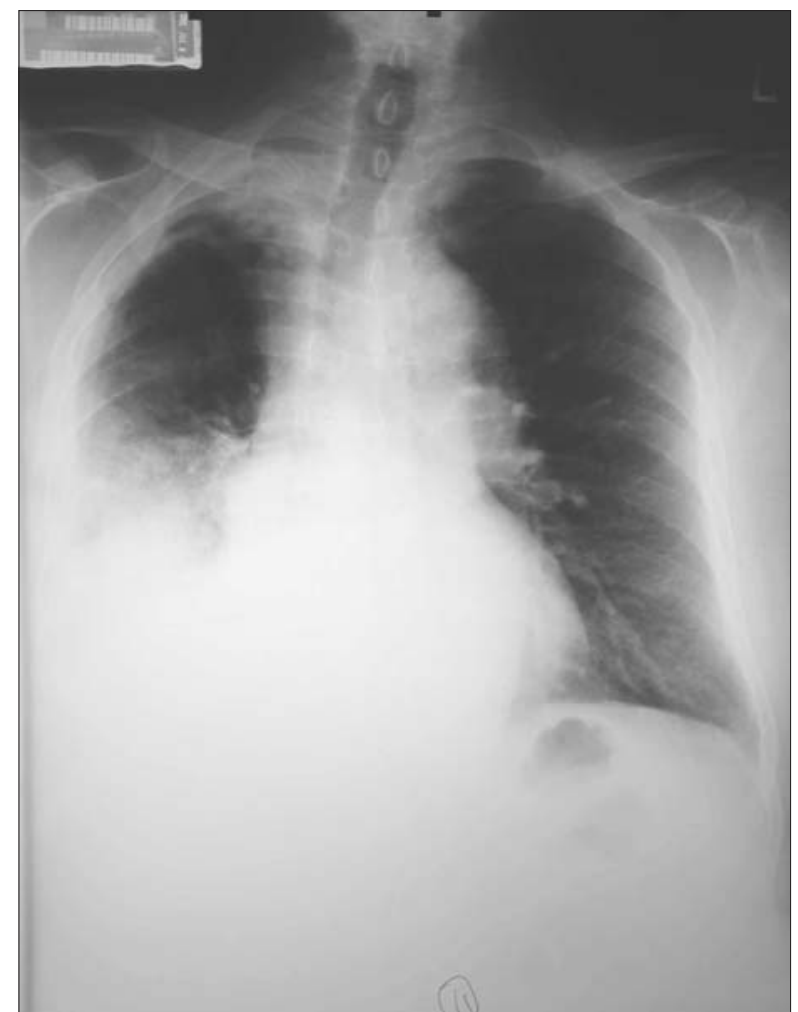

Fig. 2. - Chest radiograph at 5 days post-lobectomy showing infiltrates at right lower zone. nant cells or infective agents. Transbronchial lung biopsy revealed nodular myxoid granulation tissue polyps in alveolar spaces and ducts. Serial sections showed mild chronic inflammation and focal aggregation of foamy macrophages within the alveoli. No hyaline membranes, microorganisms or tumor cells were seen. The pathological diagnosis of bronchiolis obliterans organising pneumonia was made. Systemic corticosteroid therapy with prednisolone at a dose of $45 \mathrm{mg}$ per day (0.6 $\mathrm{mg} / \mathrm{kg} /$ day) was initiated. Dramatic clinical and radiological response was observed after 2 weeks of treatment. Symptoms of exertional dyspnoea, fever and cough gradually subsided. Chest X-ray after 2 weeks of prednisolone also showed improvement with substantial clearing of the shadows at both lungs (figure 4). The patient tolerated the steroid therapy well with no significant side effects except slight worsening in diabetic control requiring increase in dosage of oral hypoglycaemic agents. Systemic steroid was slowly tapered to prednisolone $10 \mathrm{mg} /$ day over 3 months and completely taken off at 6 months. He was regularly followed up and was last seen 1 year after cessation of steroid therapy. He remained well with no clinical or radiographic evidence of recurrence of BOOP or lung cancer.

\section{Discussion}

BOOP is a pathological diagnosis defined by the presence of buds of granulation tissue progressing from fibrin exudates to loose collagencontaining fibroblasts in the distal air spaces. It is

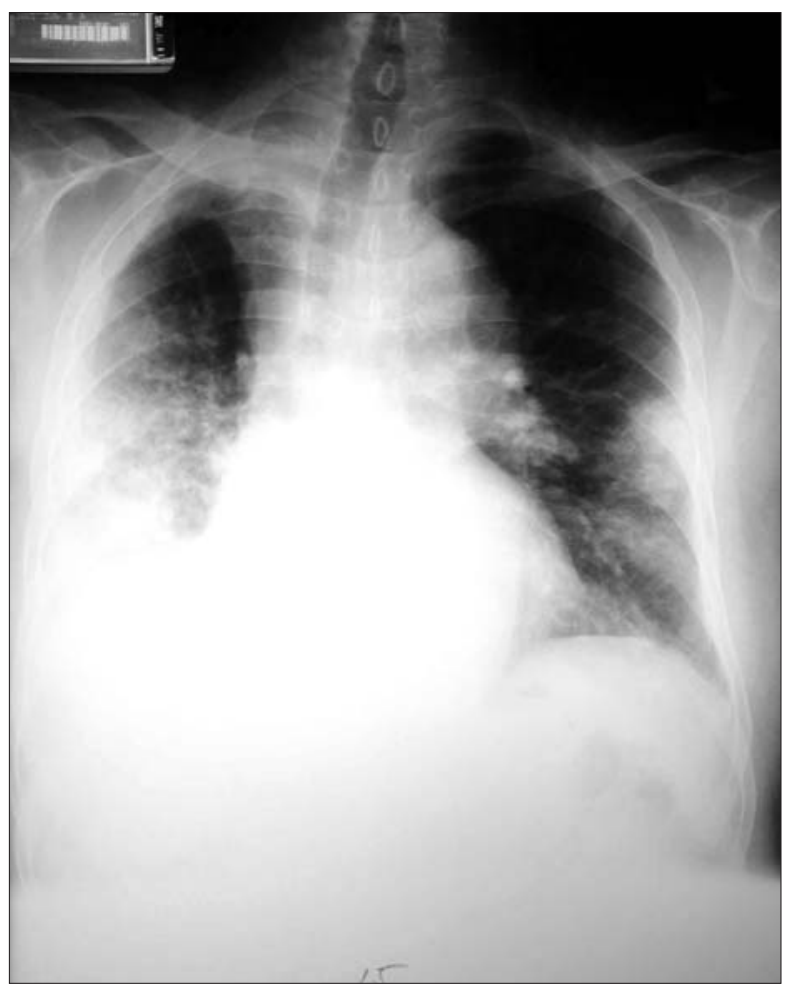

Fig. 3. - Chest radiograph at 3 weeks post-op showing deterioration with infiltrates over right lower zone, right mid zone and left lower zone. 


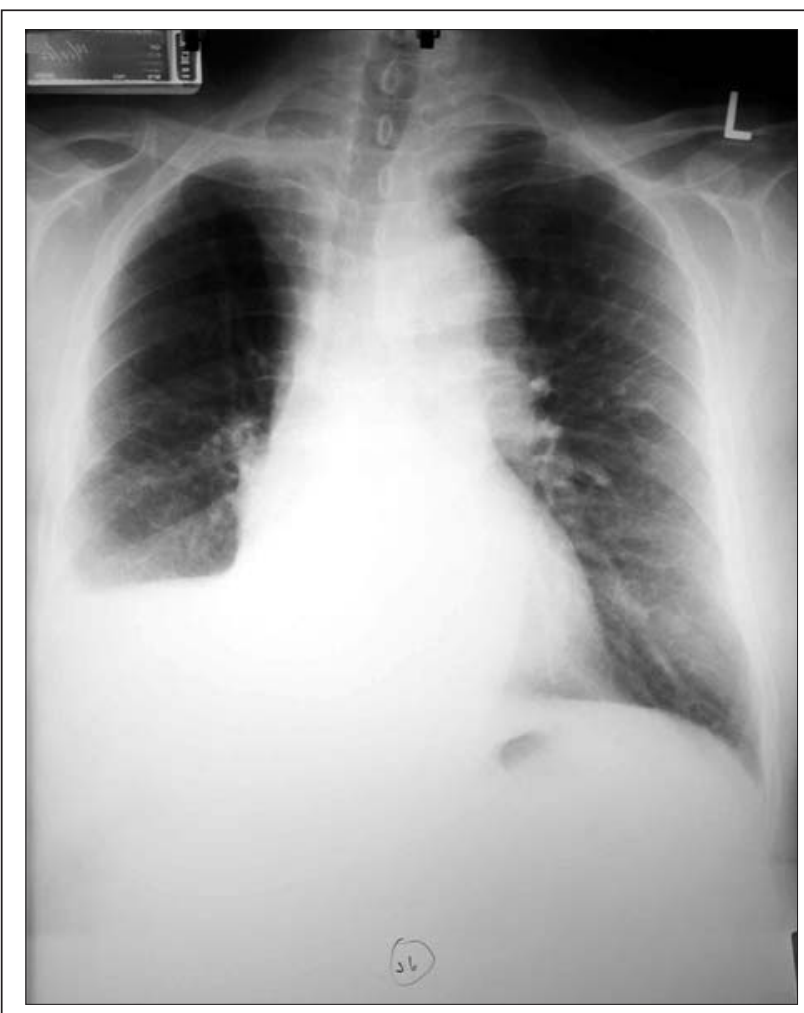

Fig. 4. - Chest radiograph 2 weeks after steroid therapy showing dramatic resolution of the pulmonary infiltrates.

often associated with buds of granulation tissue occupying the bronchiolar lumen. It reflects one type of inflammatory process after lung injury and it is not specific for any particular disorder or cause. It has been reported to be associated with some infective, inflammatory and neoplastic conditions [3] but in a large proportion of the cases, it occurs as an idiopathic form, which is now commonly referred to as 'Cryptogenic Organizing Pneumonia' (COP) [4].

The presentation of new pulmonary infiltrates after surgery for lung cancer would often prompt one to consider differential diagnoses including post-operative pneumonia, atelectasis, pulmonary embolism, and even early blood-borne or lymphangitic metastasis. However, in this case, the constellation of the relatively non-toxic clinical status, the absence of response to antibiotic therapy, the characteristic fleeting nature of the lung infiltrates and its relatively rapid evolution would suggest the possibility of BOOP or related conditions as the diagnosis. Early transbronchial or even open lung biopsy would be helpful in establishing the diagnosis. It indeed responded promptly to systemic corticosteroid therapy.

BOOP depicts one type of inflammatory process after some kind of lung injury and it is not specific for any particular disorder. The occurrence of BOOP in association with malignancy including lung cancer has been reported [2,5]. The pathological finding of organizing pneumonia adjacent to a resected lung tumor was actually common. In a study by Romero et al, adjacent organising pneumonia was found in $37 \%$ of the resected lung tumours [6]. However, they all appeared as a silent process. Arrabal Sanchez et al reported a case of adenocarcinoma found in a patient at a different part of his lung during thoracoscopic lung biopsy for a lung lesion, which was diagnosed as BOOP [5]. In a study by Mokhtari et al, among 43 cases of BOOP in association with cancer, 10 were associated with lung cancer but they all had received treatment in the form of chemotherapy and/or radiotherapy [2]. The occurrence of BOOP immediately after resection of lung cancer without adjuvant therapy, to the best of our knowledge, has never been reported in literature.

What causes the inflammatory process of BOOP in this lung cancer patient remains elusive. Although one could argue that BOOP might have just developed as an idiopathic form with the lung cancer surgery being a coincidence, the patient remained well before surgery with no flu-like symptoms which are common preceding symptoms of idiopathic BOOP. Furthermore, the pre-operative Chest radiograph and $\mathrm{CT}$ thorax did not show any early evidence of BOOP in any parts of the lung field. The patient's medications including the anaesthetic agents used have never been reported to cause BOOP. Based on these and the temporal relationship, the most likely cause of BOOP in this patient is probably still the lung cancer and/or the surgery itself. BOOP has been well reported to be associated with some auto-immune disorders, suggesting an immune-mediated mechanism $[7,8]$. In our case, the lung cancer might have presented some antigenic stimuli, which activated the host's immune system such that the inflammatory process perpetuated itself even after the surgical removal of the tumour. Alternatively, the tissue trauma itself during thoracotomy might have elicited cytokine release that triggered the inflammatory response which evolved into the process of BOOP. A closely akin condition which is also believed to be immune-mediated is post-thoracotomy acute lung injury $[9,10]$, a well recognised complication of lung resection. This is a severe form of inflammatory response of which the pathogenesis remains unclear but activation of inflammatory mediators probably plays an important role [11]. The first appearance of pneumonitis in the right lower lung field after right lower lobectomy before its extension to involve other parts of the lungs lends some support to this tissue trauma hypothesis.

In conclusion, we have reported a case of BOOP developed after lung cancer surgery. Our conjecture for the development of BOOP in this patient is that the lung cancer itself or the lung resection had excited the patient's own immune system. An inflammatory cascade with activated cells and mediators perpetuated the disease process after the initial insult causing the clinico-pathological syndrome of BOOP. In any case, we would like to remark that BOOP should be considered when new lung infiltrates develop in a patient after lung cancer surgery, especially when the clinical picture is not suggestive of an infection or thromboembolic events and the radiographic findings are characteristic of BOOP. 


\section{References}

1. Epler GR, Colby TV, McLoud TC, et al. Bronchiolitis obliterans organizing pneumonia. $N$ Engl J Med 1985; 312: 152-158.

2. Mokhtari M, Bach PB, Tietjen PA Stover DE. Bronchiolitis obliterans organizing pneumonia in cancer: a case series. Respir Med 2002; 96: 280-6.

3. Epler GR. Heterogeneity of Bronchiolitis obliterans organizing pneumonia. Curr Opin Pul Med 1998; 4: 93-97.

4. American Thoracic Society/European Respiratory Society International Multidisciplinary Consensus Classification of the Idiopathic Interstitial Pneumonias. Am J Respir Crit Care Med 2002; 165: 277-304.

5. Arrabal Sanchez R, Mongil Poce R, Benitez Domenech A, Fernandez De Rota Avecilla A, Fernandez Bermudez JL. Bronchiolitis obliterans organizing pneumonia and bronchogenic carcinoma coexisting in defferent parts of the lungs. Arch Bronchoneumol 2004; 40: 141-3.
6. Romero S, Barroso E, Rodriguez-Paniagua M, et al. Organizing pneumonia adjacent to lung cancer: frequency and clinico-pathologic features. Lung Cancer 2002; 35: 195-201.

7. Swinburn CR, Jackson GJ, Cobden I, et al. Bronchiolitis obliterans organizing pneumonia in a patient with ulcerative colitis. Thorax 1988; 43: 735-6.

8. Imasaki T, Yoshii A, Tanaka S, Ogura $\mathrm{T}$, et al. Polymyositis and Sjogren's syndrome associated with bronchiolitis obliterans organizing pneumonia. Intern Med 1996; 35: 231-5.

9. Hayes JP, Williams EA, Goldstraw P, Evans TW. Lung injury in patients following thoracotomy. Thorax 1995; 50: 990-1.

10. Williams E, Goldstraw P, Evans TW. The complications of lung resection in adults: adult respiratory distress syndrome (ARDS). Monaldi Arch Chest Dis 1996; 51: 310-5.

11. Park WY, Goodman RB, Steinberg KP, et al. Cytokine balance in the lungs of patients with acute respiratory distress syndrome. Am J Respir Crit Care Med 2001; 164: 1896-1903.

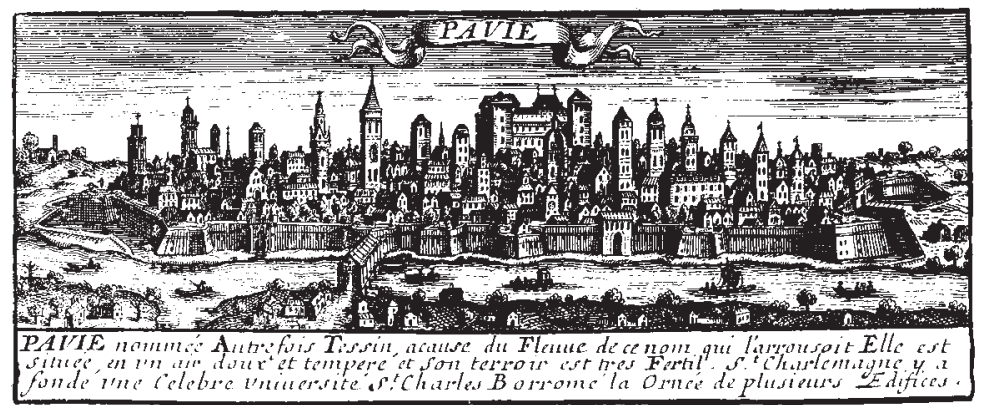

